\title{
Commentary: Is the developmentally immature immune response in paediatric sepsis a recapitulation of immune tolerance?
}

\author{
Manoj Muthukuru *t \\ Health Sciences Center, University of Washington, Seattle, WA, United States
}

Keywords: immune system, endotoxins, Toll-like receptors, inflammation, neonatal sepsis, immune tolerance

\section{A Commentary on}

OPEN ACCESS

Edited by:

Sandra Maria Blois,

Charité Medical University of

Berlin, Germany

Reviewed by:

Andrea Balogh,

Hungarian Academy of Sciences

(MTA), Hungary

Elke Winterhager,

University of

Duisburg-Essen, Germany

*Correspondence:

Manoj Muthukuru

manojm@u.washington.edu

${ }^{\dagger}$ Present address:

Manoj Muthukuru,

Holistic Health Oration LLC,

Centereach, NY, United States

Specialty section:

This article was submitted to

Immunological Tolerance and

Regulation,

a section of the journal

Frontiers in Immunology

Received: 26 October 2019 Accepted: 28 November 2019 Published: 19 December 2019

Citation: Muthukuru M (2019) Commentary: Is the developmentally immature immune response in paediatric sepsis a recapitulation of immune tolerance?

Front. Immunol. 10:2932.

doi: 10.3389/fimmu.2019.02932
Is the developmentally immature immune response in paediatric sepsis a recapitulation of immune tolerance?

by Maddux, A. B., and Douglas, I. S. (2018). Immunology 145, 1-10. doi: 10.1111/imm.12454

\section{INTRODUCTION}

This commentary is on the review article "Is the developmentally immature immune response in paediatric sepsis a recapitulation of immune tolerance?" by Maddux, A. B. and, Douglas, I. S., published in Immunology (2015) (1). In this review, the authors succinctly elaborate the immature state of the neonatal immune system and its innate immune responses, which are characteristically hypo-inflammatory. Contextually, these hypo-inflammatory responses of the neonatal immune system can be attributed to the low expression profiles of Toll-like Receptor (TLR4), the putative receptor that recognizes lipopolysaccharide (LPS) and also related to ineffective TLR signaling, which is eventually required for transcriptional activation and induction of pro-inflammatory cytokines (2). This hypo-inflammatory response is not only of the decreased production of pro-inflammatory cytokines but also of an exaggerated expression of anti-inflammatory mediators $(1,2)$.

In light of the neonatal immune response being hypo-inflammatory, however, we currently know very little as to why the prevalence, morbidity, and mortality of neonatal sepsis are significantly higher relative to pediatric sepsis affecting older children and adult sepsis (3-5). In an effort to address this apparent discrepancy with respect to bench-top findings vs. bedside outcomes, the contribution of immune or endotoxin tolerance in the neonatal immune system has been suggested (1). Since the immature neonatal immune system and the state of endotoxin tolerized immunoregulatory responses among adults are exemplified by the hypo-inflammatory response and present with striking similarities, such as low expression profiles of TLR4 and preponderance of anti-inflammatory cytokine signatures, we may arguably conclude that the overall characteristics of the neonatal immature immune responses differ from the mature immune responses in ways that are similar to the differences between endotoxin tolerance and endotoxin sensitized responses (1).

The complex molecular regulations that are associated with the induction of endotoxin tolerance are attempts to blunt the unfavorable aspects or the proverbial double-edged sword that connotes overall inflammatory responses (6); however, the downside of endotoxin tolerance also results in ineffective microbial killing and antigen presentation and appropriately inducing co-stimulatory signals for activating adaptive immune responses that could cause persistence of chronic inflammatory conditions or coexisting secondary infections during sepsis (7). These 
dampened adaptive immune responses, which are associated with endotoxin tolerance, present another dimension of confounding similarities with that of the neonatal immune system wherein, newborns not only have low amounts of circulating monocytes and dendritic cells but these cells also have lower levels of antigen-presenting MHC-II and co-stimulatory molecules, such as CD80 and CD86, compared to adults, which is indicative of the inability of the neonatal immune system to fully activate antigen-specific adaptive immune responses (2).

\section{DISCUSSION}

We must reiterate that the fundamental basis of endotoxin tolerance is exemplified by the resistance to subsequent LPS challenges after the host has been exposed to sub-lethal doses of LPS (8). Thereby, neonatal immune responses have to exhibit any of the aspects that are related to an endotoxin tolerized state, there must be prior exposure and sensitization to LPS. It is from this context that the overall immunoregulatory aspects that are associated with the induction of endotoxin tolerance are referred to as "cellular reprogramming" that defines the status of immune cells during sepsis or systemic inflammatory response syndrome (8). Computational biological methods and mathematical modeling have vigorously explored distinct network topologies that are associated with these distinct LPS exposures and the state of immune responsiveness through either LPS priming or endotoxin tolerance (9). Moreover, this state of cellular reprogramming that is associated with endotoxin tolerance is sustainable for extended period of time and therefore, these immunoregulatory aspects also demonstrate the attributes of innate memory $(10,11)$. In order for effectual tolerizing responses to partake in the overall immune responses, therefore, the ontology of the immune system should obtain instructions from the environmental signals that are predominantly derived from postnatal microbial colonization (12).

Moreover, at this juncture, we also observe that the molecular aspects of endotoxin tolerance have been extensively but exclusively investigated in adult leukocytes, either from humans or from animal models, and furthermore, these studies have established the mechanistic existence of the endotoxin tolerized states among adults after the clinical or experimental squeal of severe sepsis (8) and during localized chronic inflammatory conditions, such as periodontitis (13-15) and inflammatory bowel diseases (16). Recent investigations exploring the field of neonatal immunology have also

Abbreviations: TLR, Toll-like receptor; LPS, lipopolysaccharide.

\section{REFERENCES}

1. Maddux AB, Douglas IS. Is the developmentally immature immune response in paediatric sepsis a recapitulation of immune tolerance? Immunology. (2015) 145:1-10. doi: 10.1111/imm. 12454

2. Basha S, Surendran N, Pichichero M. Immune responses in neonates. Expert Rev Clin Immunol. (2014) 10:1171-84. doi: 10.1586/1744666X.2014.942288 critically analyzed sepsis and have associated immunoregulatory responses that are associated with immunosuppression with endotoxin tolerized states (17). However, we currently lack direct experimental evidence from clinical studies and relevant genomic analyses that can establish these distinctively altered immune responses during sepsis and associate these features with the age-related maturational states of the immune system $(18,19)$. Critical understanding of these immune and immunoregulatory functions through the prenatal, neonatal, and postnatal developmental stages have to be evaluated from the overall evolutionarily governing principles that are related to entropy; i.e., the metabolic programming during fetal development is tuned toward conservation of energy, and these aspects characteristically correlate with the immune responses that are resistant or homeostatic vs. responsive or tolerogenic (20,21). These aspects have profound clinical implications as we routinely encounter altered physiological states during pediatric vaccinations when the host immune response is primed through vaccine adjuncts. However, from the overall health restorative aspects of the host, it is currently unclear how the immune tolerizing responses should be exploited.

Therefore, on the basis of the critical interpretation of the immunoregulatory aspects that are associated with the induction of endotoxin tolerance, along with the clinical presentations of neonatal and pediatric sepsis and the lack of established studies that have neither evaluated nor established the existence of this critical endotoxin tolerance response during the early stages of the development of the immune system, I present my hypothesis: Immature immune responses of the neonatal immune system are predominantly anti-inflammatory, but the mechanistic aspects of immune or endotoxin tolerance that share some of these immunologically similar signatures of hypo-inflammatory response must be distinctly different in the neonatal immune system that begins the transition from the sterile intrauterine environment into the world of microbial colonization and sensitization.

\section{AUTHOR CONTRIBUTIONS}

The author confirms being the sole contributor of this work and has approved it for publication.

\section{FUNDING}

The publication cost of this article was supported by Holistic Health Oration LLC.

3. Hartman ME, Linde-Zwirble WT, Angus DC, Watson RS. Trends in the epidemiology of pediatric severe sepsis*. Pediatr Crit Care Med. (2013) 14:686-93. doi: 10.1097/PCC.0b013e3182917fad

4. Jaramillo-Bustamante JC, Marin-Agudelo A, Fernandez-Laverde M, BarenoSilva J, Epidemiology of sepsis in pediatric intensive care units: first Colombian multicenter study. Pediatr Crit Care Med. (2012) 13:501-8. doi: 10.1097/PCC.0b013e31823c980f 
5. Liu L, Johnson HL, Cousens S, Perin J, Scott S, Lawn JE, et al. Global, regional, and national causes of child mortality: an updated systematic analysis for 2010 with time trends since 2000. Lancet. (2012) 379:2151-61. doi: 10.1016/S0140-6736(12)60560-1

6. Janeway CA Jr. Approaching the asymptote? Evolution and revolution in immunology. Cold Spring Harb Symp Quant Biol. (1989) 54(Pt 1):1-13. doi: 10.1101/SQB.1989.054.01.003

7. Muthukuru M, Cutler CW. Antigen capture of Porphyromonas gingivalis by human macrophages is enhanced but killing and antigen presentation are reduced by endotoxin tolerance. Infect Immun. (2008) 76:477-85. doi: 10.1128/IAI.00100-07

8. Cavaillon JM, Adib-Conquy M. Bench-to-bedside review: endotoxin tolerance as a model of leukocyte reprogramming in sepsis. Crit Care. (2006) 10:233. doi: 10.1186/cc5055

9. Fu Y, Glaros T, Zhu M, Wang P, Wu Z, Tyson JJ, et al. Network topologies and dynamics leading to endotoxin tolerance and priming in innate immune cells. PLoS Comput Biol. (2012) 8:e1002526. doi: 10.1371/journal.pcbi.1002526

10. Netea MG, Joosten LA, Latz E, Mills KH, Natoli G, Stunnenberg HG, et al. Trained immunity: a program of innate immune memory in health and disease. Science. (2016) 352:aaf1098. doi: 10.1126/science.aaf1098

11. Seeley JJ, Baker RG, Mohamed G, Bruns T, Hayden MS, Deshmukh $\mathrm{SD}$, et al. Induction of innate immune memory via microRNA targeting of chromatin remodelling factors. Nature. (2018) 559:114-9. doi: 10.1038/s41586-018-0253-5

12. Kollmann TR, Kampmann B, Mazmanian SK, Marchant A, Levy O. Protecting the newborn and young infant from infectious diseases: lessons from immune ontogeny. Immunity. (2017) 46:350-63. doi: 10.1016/j.immuni.2017.03.009

13. Muthukuru M. Towards endotoxin tolerance or not? Implications from localized odontogenic periodontitis to systemic septic shock syndrome. Med Hypotheses. (2019) 134:109418. doi: 10.1016/j.mehy.2019.109418

14. Muthukuru M, Cutler CW. Upregulation of immunoregulatory Src homology 2 molecule containing inositol phosphatase and mononuclear cell hyporesponsiveness in oral mucosa during chronic periodontitis. Infect Immun. (2006) 74:1431-5. doi: 10.1128/IAI.74.2.1431-1435.2006
15. Muthukuru M, Jotwani R, Cutler CW. Oral mucosal endotoxin tolerance induction in chronic periodontitis. Infect Immun. (2005) 73:687-94. doi: 10.1128/IAI.73.2.687-694.2005

16. Vamadevan AS, Fukata M, Arnold ET, Thomas LS, Hsu D, Abreu MT. Regulation of Toll-like receptor 4-associated MD-2 in intestinal epithelial cells: a comprehensive analysis. Innate Immun. (2010) 16:93-103. doi: $10.1177 / 1753425909339231$

17. Hibbert JE, Currie A, Strunk T. Sepsis-induced immunosuppression in neonates. Front Pediatr. (2018) 6:357. doi: 10.3389/fped.2018.00357

18. Wynn JL, Cvijanovich NZ, Allen GL, Thomas NJ, Freishtat RJ, Anas N, et al. The influence of developmental age on the early transcriptomic response of children with septic shock. Mol Med. (2011) 17:1146-56. doi: 10.2119/molmed.2011.00169

19. Raymond SL, Lopez MC, Baker HV, Larson SD, Efron PA, Sweeney $\mathrm{TE}$, et al. Unique transcriptomic response to sepsis is observed among patients of different age groups. PLoS ONE. (2017) 12:e0184159. doi: 10.1371/journal.pone.0184159

20. Vachharajani V, McCall CE. Epigenetic and metabolic programming of innate immunity in sepsis. Innate Immun. (2019) 25:267-79. doi: 10.1177/1753425919842320

21. Kan B, Michalski C, Fu H, Au HHT, Lee K, Marchant EA, et al. Cellular metabolism constrains innate immune responses in early human ontogeny. Nat Commun. (2018) 9:4822. doi: 10.1038/s41467-018-07215-9

Conflict of Interest: The author declares that the research was conducted in the absence of any commercial or financial relationships that could be construed as a potential conflict of interest.

Copyright (c) 2019 Muthukuru. This is an open-access article distributed under the terms of the Creative Commons Attribution License (CC BY). The use, distribution or reproduction in other forums is permitted, provided the original author(s) and the copyright owner(s) are credited and that the original publication in this journal is cited, in accordance with accepted academic practice. No use, distribution or reproduction is permitted which does not comply with these terms. 